\title{
Isolation au postglaciaire d'un bassin côtier près de Kuujjuaraapik-Whapmagoostui, en Hudsonie (Québec) : une analyse biostratigraphique diatomifère Postglacial isolation of a coastal basin near Kuujjuaraapik-Whapmagoostui, Hudsonie: A diatom biostratigraphical investigation Postglaziale Isolation eines küstennahen Beckens nahe Kuujjuaraapik-Whapmagoostui, Hudsonie: Eine Diatomeenanalyse.
}

\author{
Émilie Saulnier-Talbot et Reinard Pienitz
}

Volume 55, numéro 1, 2001

URI : https://id.erudit.org/iderudit/005662ar

DOI : https://doi.org/10.7202/005662ar

Aller au sommaire du numéro

Éditeur(s)

Les Presses de l'Université de Montréal

ISSN

0705-7199 (imprimé)

1492-143X (numérique)

Découvrir la revue

Citer cet article

Saulnier-Talbot, É. \& Pienitz, R. (2001). Isolation au postglaciaire d'un bassin côtier près de Kuujjuaraapik-Whapmagoostui, en Hudsonie (Québec) : une analyse biostratigraphique diatomifère. Géographie physique et Quaternaire, 55(1), 63-74. https://doi.org/10.7202/005662ar

\section{Résumé de l'article}

En vue de retracer les phases d'isolation postglaciaire d'un lac côtier situé près de Kuujjuaraapik-Whapmagoostui (baie d'Hudson), le contenu diatomifère d'une carotte de sédiments a été analysé. La séquence sédimentaire recueillie dans le lac Kachishayoot démontre trois unités de changements paléoenvironnementaux provoqués par le relèvement isostatique postglaciaire. Ces unités se distinguent entre elles par une sédimentologie et des assemblages fossiles de diatomées contrastants. Le passage de l'argile marine à la gyttja et l'évolution des assemblages diatomifères successivement dominés par des espèces poly-mésohalobes, oligohalobes, puis halophobes, démontrent bien les effets de la salinité décroissante sur le milieu aquatique. De plus, les valeurs décroissantes d'alcalinité calculées par l'intermédiaire d'une fonction de transfert (basée sur les assemblages de diatomées) reflètent le passage d'un environnement riche à un environnement pauvre en nutriments lors de l'isolation du bassin lacustre des eaux de la Mer postglaciaire de Tyrrell. Le recyclage graduel de ses eaux a ainsi éliminé les nutriments et les ions majeurs (carbone inorganique dissous, $\mathrm{Ca}, \mathrm{Mg}$ ) issus du délavement du till et des argiles marines. La datation, par spectrométrie de masse à l'aide d'un accélérateur de particules (SMA) de matériel provenant de la carotte a permis d'évaluer le taux de sédimentation et d'estimer la durée des trois différentes étapes de la succession lacustre dans le bassin du lac Kachishayoot (conditions marines de 5400-4500 ans cal. BP, phase d'isolation entre 4500-1600 ans cal. BP et la phase lacustre depuis 1600 ans cal. BP). Cette étude biostratigraphique fournit aussi des indices quant à l'évolution du paysage dans le sud de l'Hudsonie par suite du retrait de la Mer postglaciaire de Tyrrell, il y a environ 5000 ans.
Tous droits réservés @ Les Presses de l'Université de Montréal,2001
Ce document est protégé par la loi sur le droit d'auteur. L’utilisation des services d’Érudit (y compris la reproduction) est assujettie à sa politique d'utilisation que vous pouvez consulter en ligne.

https://apropos.erudit.org/fr/usagers/politique-dutilisation/ 


\title{
ISOLATION AU POSTGLACIAIRE D'UN BASSIN CÔTIER PRĖS DE KUUJJUARAAPIK-WHAPMAGOOSTUI, EN HUDSONIE (QUÉBEC) : UNE ANALYSE BIOSTRATIGRAPHIQUE DIATOMIFĖRE
}

\author{
Émilie SAULNIER-TALBOT* et Reinard PIENITZ*, Laboratoire de paléolimnologie et paléoécologie, Centre d'études \\ nordiques et Département de géographie, Université Laval, Québec G1K 7P4.
}

RÉSUMÉ En vue de retracer les phases d'isolation postglaciaire d'un lac côtier situé près de Kuujjuaraapik-Whapmagoostui (baie d'Hudson), le contenu diatomifère d'une carotte de sédiments a été analysé. La séquence sédimentaire recueillie dans le lac Kachishayoot démontre trois unités de changements paléoenvironnementaux provoqués par le relèvement isostatique postglaciaire. Ces unités se distinguent entre elles par une sédimentologie et des assemblages fossiles de diatomées contrastants. Le passage de l'argile marine à la gyttja et l'évolution des assemblages diatomifères successivement dominés par des espèces poly-mésohalobes, oligohalobes, puis halophobes, démontrent bien les effets de la salinité décroissante sur le milieu aquatique. De plus, les valeurs décroissantes d'alcalinité calculées par l'intermédiaire d'une fonction de transfert (basée sur les assemblages de diatomées) reflètent le passage d'un environnement riche à un environnement pauvre en nutriments lors de l'isolation du bassin lacustre des eaux de la Mer postglaciaire de Tyrrell. Le recyclage graduel de ses eaux a ainsi éliminé les nutriments et les ions majeurs (carbone inorganique dissous, $\mathrm{Ca}, \mathrm{Mg}$ ) issus du délavement du till et des argiles marines. La datation, par spectrométrie de masse à l'aide d'un accélérateur de particules (SMA) de matériel provenant de la carotte a permis d'évaluer le taux de sédimentation et d'estimer la durée des trois différentes étapes de la succession lacustre dans le bas$\sin$ du lac Kachishayoot (conditions marines de 5400-4500 ans cal. BP, phase d'isolation entre 4500-1600 ans cal. BP et la phase lacustre depuis 1600 ans cal. BP). Cette étude biostratigraphique fournit aussi des indices quant à l'évolution du paysage dans le sud de l'Hudsonie par suite du retrait de la Merpostglaciaire de Tyrrell, il y a environ 5000 ans.
ABSTRACT Postglacial isolation of a coastal basin near Kuujjuaraapik-Whapmagoostui, Hudsonie: A diatom biostratigraphical investigation. In order to retrace the successive postglacial isolation phases of a coastal lake located near Kuujjuaraapik-Whapmagoostui (eastern Hudson Bay), the diatom assemblages of a sediment core were analysed. The sedimentary sequence collected from Kachishayoot Lake shows three zones corresponding to paleoenvironmental changes brought about by isostatic rebound. Each zone is characterized by a contrasting sedimentology and fossil diatom assemblages. The transition from marine clays to gyttja and the evolution of the diatom assemblages, successively dominated by poly-mesohalobian, oligohalobian and finally halophobous species, documents the overall trend of decreasing salinity in the lake. Diatominferred alkalinity also decreased, most likely reflecting the change from a nutrient-rich to a nutrient-poor environment as the basin was isolated from the waters of the postglacial Tyrrell Sea and gradually purged of nutrients and major ions (dissolved inorganic carbon, $\mathrm{Ca}$, $\mathrm{Mg}$ ) from the carbonate weathering of glacial tills and marine clays. Chronological references obtained through AMS dating made it possible to evaluate sedimentation rates and the duration of the three different stages (marine conditions from 5400-4500 cal yr BP, isolation phase between $4500-1600$ cal yr BP and modern lake phase since $1600 \mathrm{cal}$ yr BP) of lake succession in the Kachishayoot Lake basin. This biostratigraphic study yields information about the evolution of the landscape in the southeastern Hudson Bay area following the retreat of the postglacial Tyrrell Sea about 5000 years ago.
ZUSAMMENFASSUNG Postglaziale Isolation eines küstennahen Beckens nahe Kuujjuaraapik-Whapmagoostui, Hudsonie: Eine Diatomeenanalyse. Die fossilen Diatomeen eines Sedimentkerns wurden untersucht, um den postglazialen Isolationsprozeß eines küstennahen Sees zu rekonstruieren. Die Sedimente stammen aus einem Becken im Gebiet der östlichen Hudson Bay, etwa $10 \mathrm{~km}$ nördlich von Kuujjuaraapik-Whapmagoostui gelegen. Basierend auf sedimentologischen und biostratigraphischen Analysen konnten drei Entwicklungsphasen differenziert werden: Der Übergang vom Meeresbecken zum Süßwassersee zeigt sich in der Abfolge Meereston-Gyttja und der damit einhergehenden Sukzession poly-mesohalober, oligohalober und schließlich halophober Gattungen, bedingt durch abnehmenden Salzgehalt. Die von einer Diatomeentransferfunktion hergeleiteten Alkalinitätswerte weisen ebenfalls auf den Isolationsproze $B$ des Beckens vom marinen Einfluß des Tyrellmeers hin. Die zeitliche Abfolge der Umweltveränderungen wurde mittles AMS Datierungen von Pflanzenmaterial ermittlet. Auf diese Weise ergab sich die Dauer jeder Entwicklungsphase des Kachishayoot-Sees wie folgt : eine Meerphase von etwa 54004500 cal. Jahre v.u.Z., eine Isolationsphase zwischen etwa 4500-1600 cal. Jahre v.u.Z. und seit etwa 1600 cal. Jahre v.u.Z., Süßwasserbedingungen. Diese biostratigraphische Untersuchung liefert Informationen über die Änderung der Landschaften im Gebiet der südöstlichen Hudson Bay nach dem Rückzug des postglazialen Tyrrellmeers vor etwa 5000 Jahren. 


\section{INTRODUCTION}

Depuis la fonte de l'Inlandsis laurentidien dans le nord et le moyen-nord québécois, la région de la Jamésie-Hudsonie a connu une succession de paysages différents. Le lac proglaciaire Ojibway s'est développé vers 12000 BP au moment où les glaciers se retiraient et a probablement atteint sa limite nord-est à l'embouchure de la Grande Rivière de la Baleine (Vincent et Hardy, 1977 ; Veillette, 1994). Vers 7900 BP, lorsque les glaciers d'Hudson et du Nouveau-Québec se sont séparés à la latitude du détroit d'Hudson ( $\mathrm{ca} .63^{\circ} \mathrm{N}, 70^{\circ} \mathrm{O}$ ), la région fut envahie par la Mer postglaciaire de Tyrrell (Bilodeau et al., 1990).

En réponse au relèvement isostatique postglaciaire, la côte est de la baie d'Hudson a connu un des taux d'émersion des terres les plus élevés au monde (environ $275 \mathrm{~m}$ depuis la déglaciation, se poursuivant aujourd'hui à un rythme de $10 \mathrm{~mm} . \mathrm{a}^{-1}$; Allard et Tremblay, 1983). Le paysage côtier hudsonien a donc subi durant l'Holocène de grandes modifications par suite de la régression marine et de la migration progressive de la ligne de rivage vers le large. Par la suite, plusieurs lacs se sont formés le long de la côte.

Les lacs sont une composante majeure du paysage subarctique québécois. De par leur capacité à préserver les marqueurs de leur histoire dans les séquences sédimentaires, ils sont des lieux privilégiés pour l'étude des conditions environnementales du passé. Grâce à leurs enveloppes siliceuses, les diatomées (classe Bacillariophyta) comptent parmi les microfossiles les mieux préservés dans les sédiments lacustres et marins. Plusieurs études diatomifères ont déjà démontré leur utilité à reconstituer la dynamique des paléoenvironnements côtiers (Kjemperud, 1981 ; Lie et al., 1983 ; Palmer et Abbott, 1986 ; Stabell, 1985 ; Zong, 1997a ; Seppä et Tikkanen, 1998 ; Campeau et al., 1999a et b, 2000). Au Québec, par contre, la seule étude comparable est celle de Pienitz et al. (1991), dans la région de Kuujjuaq, qui a, par l'intermédiaire d'une analyse diatomifère, démontré la régression marine de la côte ungavienne.

Cet article vise à mieux comprendre la formation de lacs dans le contexte d'une côte en émersion rapide et à préciser l'évolution des bassins d'eau douce dans la région subarctique québécoise, par une analyse biostratigraphique diatomifère et la reconstitution des variations historiques du taux d'alcalinité de l'eau. Cette approche n'a auparavant jamais été appliquée dans la région sud-est de la baie d'Hudson.

\section{SITE D'ÉTUDE}

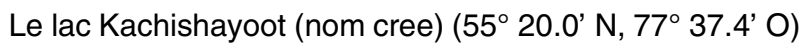
se situe à $3 \mathrm{~km}$ de la côte est de la baie d'Hudson, près du village de Kuujjuaraapik-Whapmagoostui, au Québec nordique (fig. 1). Il repose sur des granites et des gneiss du Bouclier canadien à une altitude d'environ $100 \mathrm{~m}$. Le relief environnant, érodé par l'avancée des glaciers, est peu accidenté. Une grande partie du paysage côtier est composée de plateaux silteux légèrement inclinés et de plages sablonneuses reposant sur la roche en place (Bégin et al., 1993).

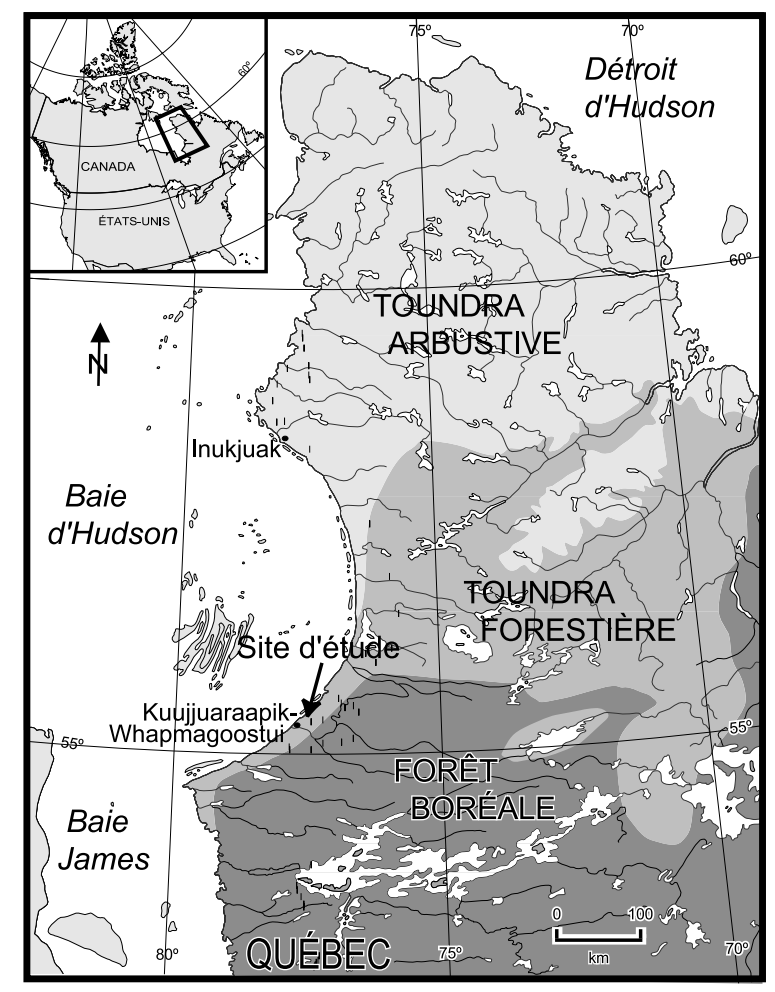

FIGURE 1. Localisation du site d'étude et principales zones de végétation.

Location of the study site and major vegetation zones (Boreal Forest, Forest Tundra and Shrub Tundra).

Le lac Kachishayoot (fig. 2) est un lac polymictique oligotrophe peu profond $(Z \max =5 \mathrm{~m}$ ), avec une température de l'eau hivernale d'environ $4^{\circ} \mathrm{C}$ et variant entre 13 et $20^{\circ} \mathrm{C}$ en été (Gibson et al., 2001). Son bassin est d'une superficie d'environ 29,5 ha et son bassin de drainage de 210 ha (tabl. I).

La majeure partie du bassin versant (environ $70 \%$ ) est recouverte d'une mince couche de cryptogames, incluant des mousses et des lichens (principalement Cladina stellaris), reposant sur la roche mère. Le reste du territoire est peuplé par l'épinette noire et blanche (Picea mariana, $P$. glauca), le saule (Salix spp.), le bouleau glanduleux (Betula glandulosa), l'aulne crispé (Alnus crispa), ainsi que par plusieurs espèces de plantes basses dont l'assemblage est dominé par la camarine noire (Empetrum nigrum) et le thé du Labrador (Ledum groenlandicum).

L'Hudsonie est actuellement caractérisée par un climat continental subarctique, avec des vents dominants d'été provenant de l'ouest et une saison sans gel d'une durée d'environ 90 jours (Richard, 1979). La moyenne annuelle des températures enregistrées à Kuujjuaraapik-Whapmagoostui entre 1968 et 1998 est de $-4,54{ }^{\circ} \mathrm{C}$, le mois de juillet étant le plus chaud (Environnement Canada, 1999). Les précipitations annuelles sont d'environ $660 \mathrm{~mm}$, dont $40 \%$ tombent sous forme de neige (Bégin et al., 1993). En raison de la rigueur du climat côtier, une bande de toundra forestière s'étend le long 
FIGURE 2. Carte du bassin versant du lac Kachishayoot montrant la topographie et les zones de végétation simplifiées, ainsi que la localisation du lac par rapport aux îles Manitounuk.

Map of the drainage basin of Kachishayoot Lake showing the topography and simplified vegetation zones, as well as the location of the site in relation to the Manitounuk Islands.

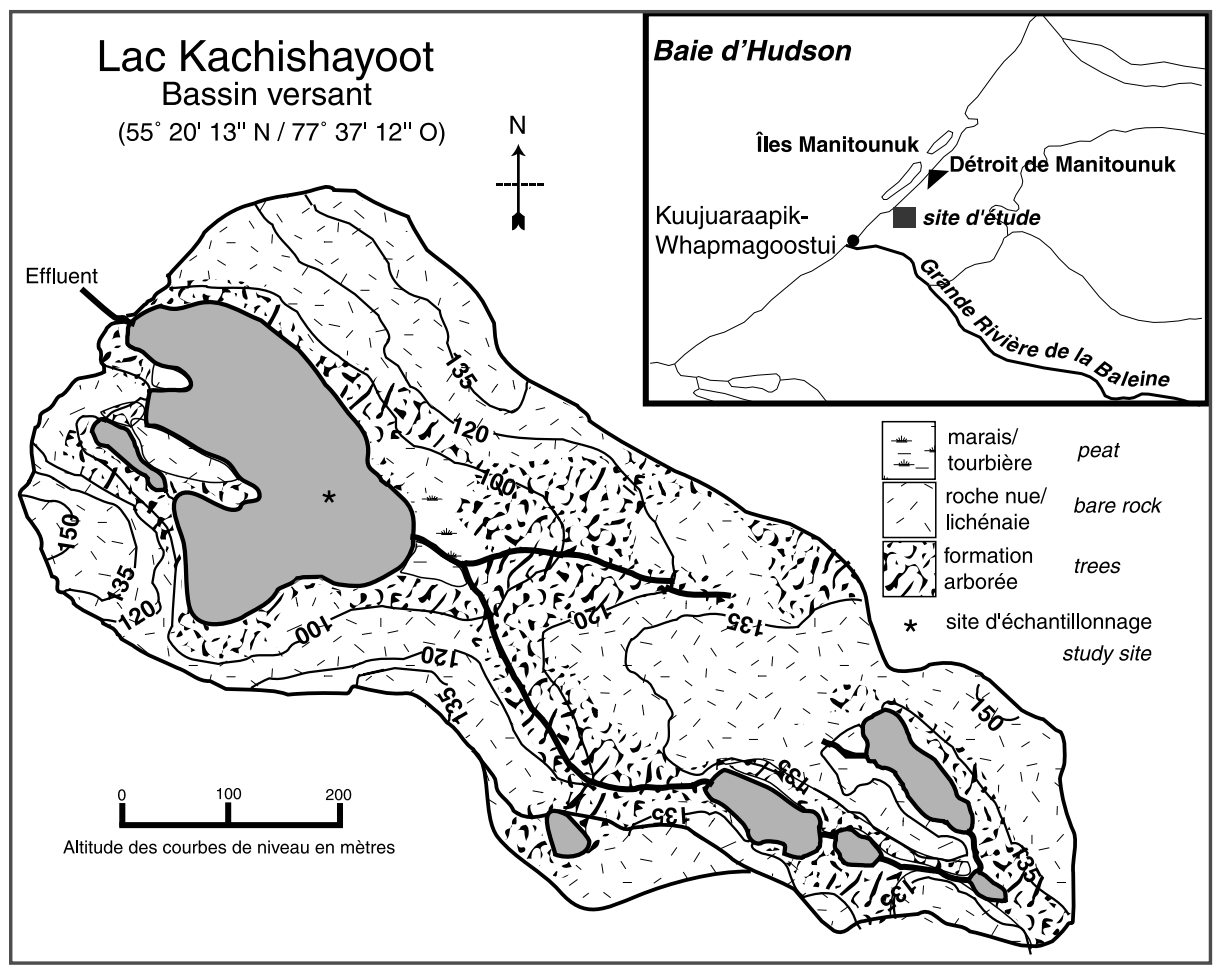

TABLEAU I

Sommaire des renseignements morphométriques et topographiques du lac Kachishayoot ( $\left.55^{\circ} 20.0^{\prime} \mathrm{N}, 77^{\circ} 37.4^{\prime} \mathrm{O}\right)$

Profondeur maximale enregistrée $(\mathrm{m})$

Surface $\left(\mathrm{km}^{2}\right)$

Longueur totale de la ligne de rivage $(\mathrm{km})$

Longueur maximale $(\mathrm{m})$

0,295

Largeur maximale $(\mathrm{m})$

Surface couverte par le bassin versant $\left(\mathrm{km}^{2}\right)$

Rapport surface du lac /surface du bassin versant

Altitude $(\mathrm{m})$

Élévation du relief environnant $(\mathrm{m})$

Distance de la baie d'Hudson $(\mathrm{km})$

de la côte (fig. 1). Elle est caractérisée par un couvert forestier sporadique limité aux milieux protégés et par une zone de pergélisol discontinu (Payette, 1983 ; Allard et Séguin, 1987).

\section{MATÉRIEL ET MÉTHODES}

\section{MÉTHODES DE TERRAIN}

Le prélèvement des carottes de sédiments s'est effectué au mois de juin 1998. Une carotte courte d'une longueur de $36,5 \mathrm{~cm}$ fut prélevée à l'aide d'un carottier à gravité de type K-B (Kajak-Brinkhurst), cette technique de carottage permettant une meilleure conservation de la séquence des sédiments de surface. Une carotte longue de $80 \mathrm{~cm}$ fut extraite avec un carottier à piston (modifié, de type Livingstone). Les deux carottes furent prélevées près du point le plus profond du lac (environ $5 \mathrm{~m}$ ). L'extraction des macrofossiles destinés à la datation par spectrométrie de masse accélérée (SMA) et le sous-échantillonnage des carottes à intervalles de $0,5 \mathrm{~cm}$ ont été effectués sur le terrain. Les échantillons ont été rangés dans des sacs de plastique (sédiments) et dans des fioles de verre (macrofossiles) et gardés au réfrigérateur $\left(a ̀{ }^{\circ} \mathrm{C}\right.$ ) avant d'être traités en laboratoire.

\section{DATATIONS}

Au total, quatre datations SMA ont été obtenues au laboratoire Isotrace de l'Université de Toronto à partir du matériel végétal provenant de la carotte longue (tabl. II). Les extrapolations pour le calcul du taux de sédimentation ont été faites à partir des datations TO-7592 et TO-7594. Les datations TO-7591 et TO-7593 ont été rejetées en raison d'une possibilité d'erreurs trop élevées ( \pm 500 et $\pm 150{ }^{14} \mathrm{C}$ ).

\section{MÉTHODES DE LABORATOIRE}

Le contenu en eau a été déterminé en séchant les échantillons à $65^{\circ} \mathrm{C}$ pendant 24 heures. Une combustion subséquente à $550^{\circ} \mathrm{C}$ pendant 2 heures a permis de mesurer la perte au feu (PAF), c'est-à-dire le contenu en matière organique des sédiments.

L'extraction des fossiles de diatomées a été faite suivant les techniques standard de digestion à l'acide nitrique/sulfurique (Pienitz et al., 1995 ; Wilson et al., 1996). Les échantillons ont été chauffés pendant 2 heures (ca. $80^{\circ} \mathrm{C}$ ), puis décantés plusieurs fois avec de l'eau distillée de façon à ramener le $\mathrm{pH}$ des solutions siliceuses à près de 7 . Différentes concentrations de chacun des échantillons ont été déposées sur des lamelles et laissées à sécher avant d'être montées sur des lames de microscope à l'aide d'une résine permanente (Naphrax, indice de réfraction 1,74). 
TABLEAU II

Datations SMA calibrées provenant de la carotte du lac Kachishayoot, Québec

\begin{tabular}{ccccc}
\hline numéro de laboratoire & profondeur $(\mathrm{cm})$ & matériel daté & âge $\left(\mathrm{ans}{ }^{14} \mathrm{C}\right.$ BP) & âge (ans cal. BP) \\
\hline TO-7591 & 11,0 & aiguille de Picea & mariana & 2027 \\
TO-7592 & $34,0-34,5$ & bois $^{*}$ & $1420 \pm 80$ & 1334 \\
TO-7593 & 50,5 & frag. de plante herbacée & $4340 \pm 150$ & 4918 \\
TO-7594 & 79,0 & brindille de Ledum & $4640 \pm 70$ & 5378 \\
\hline
\end{tabular}

*dates retenues pour l'extrapolation de l'âge de chaque niveau analysé

Les dates calibrées en années du calendrier avant 1950 (ans cal. BP) sont le point milieu de l'écart de probabilité des dates calculées à I'aide du logiciel CALIB v. 3.03 (Stuiver et Reimer, 1993). Les datations SMA ont été obtenues au laboratoire Isotrace de l'Université de Toronto, Canada.

Au total, trente-huit comptages sur la carotte longue et quinze sur la carotte courte (à tous les $2,5 \mathrm{~cm}$ en général) ont été effectués au microscope photonique Leica DMRB à un grossissement de 1000X sous immersion. Un minimum de 500 valves par échantillon a été dénombré afin de caractériser l'assemblage diatomifère de chaque niveau, à l'exception de quelques niveaux de la partie inférieure de la carotte longue qui montraient une très faible concentration de diatomées. À ces niveaux, toutes les valves qui se trouvaient sur la lamelle ont été comptées. Une variété de références taxonomiques a été consultée (Barber et Haworth, 1981 ; Krammer et Lange-Bertalot, 1986, 1988, 1991a et b ; Snoeijs, 1993 ; Snoeijs et Vilbaste, 1994 ; Snoeijs et Potapova, 1995 ; Snoeijs et Kasperoviciene, 1996 ; Snoeijs et Balashova, 1998 ; Witkowski, 1994 ; Campeau et al., 1999a), mais l'identification a principalement été basée sur Fallu et al., 2000, par souci de conformité taxonomique avec les travaux déjà effectués dans la région.

Afin de travailler avec un seul ensemble stratigraphique, les données spécifiques de la carotte courte et de la carotte longue ont été combinées. La séquence sédimentaire complète a été établie en identifiant les niveaux correspondants dans les stratigraphies des diatomées et dans les données de perte au feu et du contenu en eau.

\section{ANALYSE DES DONNÉES ET MÉTHODES STATISTIQUES}

L'abondance relative des espèces de diatomées les plus communes (c'est-à-dire celles représentant au moins $5 \%$ dans au moins un niveau) a été déterminée à l'aide des logiciels TILIA (version 2.00, Grimm, 1991) et TILIAGRAPH (version 1.25, Grimm, 1991-1993). Le logiciel CONISS (Grimm, 1987) a permis de délimiter, par analyse de groupement, les différentes zones stratigraphiques de diatomées. La longueur de corde a été choisie comme coefficient de dissimilarité afin de faire ressortir les espèces sub-dominantes tout en amoindrissant le poids des espèces dominantes (Overpeck et al., 1985).

Le taux de changement entre assemblages de diatomées stratigraphiquement adjacents dans la carotte a été calculé à l'aide du logiciel RATEPOL (J.M. Line et H.J.B. Birks,
1994, programme non publié), en utilisant les déviations de la mesure de corde au carré moyenne (squared chord distance) comme coefficient de dissimilarité. Les échantillons à plus haute résolution que la moyenne ont été supprimés de l'analyse afin d'assurer que le taux de changement soit interpolé à résolution constante (à intervalles de 132 ans, tel que calculé par le programme à partir des datations SMA) et afin de minimiser les distorsions attribuables aux variations de résolution dans l'analyse de la carotte.

Un modèle d'inférence a été appliqué aux données fossiles afin de reconstituer quantitativement les variations d'alcalinité survenues dans le passé. Le modèle est basé sur des analyses statistiques multivariées reflétant la répartition des assemblages actuels de diatomées dans une série de 63 lacs répartis le long d'un transect latitudinal traversant quatre zones de végétation du Québec-Labrador (Fallu et al., 2000). II montre une bonne relation entre les assemblages diatomifères et l'alcalinité, avec un Jacknife $r^{2}$ de 0,63 et une racine de l'erreur quadratique moyenne de prévision (RMSEP) de $0,25 \log$ (alcalinité +1$)\left(\mu e \mathrm{I}^{-1}\right.$ ) (Fallu et al., 2000).

Les reconstitutions quantitatives ont été établies à l'aide du logiciel WA-PLS (version 1.0, Juggins et ter Braak, 1992). Ce dernier utilise une méthode de pondération basée sur un modèle à réponse unimodale dont l'optimum est estimé par une analyse des moyennes pondérées (ter Braak et Smilauer, 1998). Les reconstitutions statistiques n'ont pas été appliquées aux huit premiers niveaux inférieurs de la carotte longue, vu le manque de similarité $(0,0 \%-7,9 \%)$ entre la microflore de l'ensemble de calibration et la microflore fossile de ces niveaux. Un modèle de reconstitution de la salinité aurait été idéal pour confirmer quantitativement les observations qualitatives, mais un tel modèle n'existe pas encore pour notre région ou pour une région similaire. Le modèle du Labrador a été adopté pour la présente étude, étant donné qu'il a été conçu pour la même région géologique (Bouclier canadien) et climatique (subarctique) et qu'il existe une certaine corrélation entre les facteurs reliés à la salinité et l'alcalinité (Fallu et al. 2000).

Des techniques statistiques d'évaluation de similitude ont été appliquées afin de vérifier la compatibilité entre l'ensemble fossile et l'ensemble de calibration. Le premier test d'analogie 
a été effectué à l'aide du logiciel ANALOG (H.J.B. Birks et J.M. Line, programme non publié). II a permis de déterminer le taux minimal de confiance du modèle et de calculer la limite acceptable de dissimilarité entre l'ensemble fossile et l'ensemble de calibration. La mesure de corde élevée au carré a été utilisée comme coefficient de dissimilarité (Overpeck et al., 1985) et les intervalles de confiance ont été basés sur la moyenne de celle-ci à l'intérieur de l'ensemble de calibration (Laird et al., 1998). II a ensuite été possible d'évaluer le degré de compatibilité entre les assemblages fossiles recensés dans la carotte et l'ensemble de calibration. Les échantillons fossiles situés au-dessus de la limite de confiance de $95 \%$ sont considérés comme n'ayant pas d'analogie dans le modèle de calibration, tandis que les échantillons situés entre 75 et $95 \%$ sont classés comme ayant des analogies pauvres (Laird et al., 1998; Laing et al., 1999b).

Les données ont aussi subi un test d'adéquation (Goodness-of-fit) qui a permis de déterminer, à l'aide d'une analyse canonique de correspondance (ACC), le taux de fiabilité avec lequel le modèle de calibration pouvait estimer la variable environnementale de chaque échantillon de la carotte. L'ACC, contrainte à une seule variable explicative (alcalinité), a été faite sur l'ensemble d'échantillons modernes, les échantillons fossiles ayant été mis en mode passif en vue de calculer leur distance résiduelle au carré (DRC) par rapport à l'axe 1 (la variable environnementale). Les DRC étant déphasées (skewed), elles ont subi une transformation logarithmique afin d'atteindre approximativement la normalité. Les intervalles de confiance de $95 \%$ ont été calculés à partir des ensembles résiduels transformés. Les échantillons fossiles qui se retrouvaient à l'intérieur de ces intervalles de $95 \%$ ont été considérés comme ayant une bonne adéquation avec la variable en question (Birks et al., 1990).

Finalement, le test de l'importance de la variable reconstituée consiste à appliquer une analyse de correspondance (AC) aux valeurs reconstituées afin de vérifier si la variable environnementale du modèle choisi sert à expliquer une part importante de la variation dans les assemblages fossiles de diatomées. Les résultats de cette analyse ne permettent toutefois pas de dire si les valeurs reconstituées sont fiables ou non. L'AC est une technique d'ordination non contrainte qui maximise la dispersion des échantillons basée sur leurs assemblages de diatomées (ter Braak, 1995). Les échantillons sont disposés dans le diagramme d'ordination en fonction de leurs assemblages d'espèces de diatomées, avec l'axe 1 représentant le plus long gradient de variation dans les assemblages diatomifères. Une relation étroite entre la variable reconstituée et les « pointages » (scores) de l'axe 1 de l'AC indique qu'une grande part de cette variation peut être expliquée par la variable en question (Laing et al., 1999b). Les pointages de l'axe ont été comparés aux valeurs d'alcalinité calculées pour chaque échantillon de la séquence sédimentaire.

\section{RÉSULTATS}

\section{STRATIGRAPHIE DES SÉDIMENTS}

La carotte longue présente une séquence sédimentaire non perturbée mesurant $80 \mathrm{~cm}$ de longueur. Elle est compo-
TABLEAU III

Sédimentologie de la séquence prélevée dans le lac Kachishayoot

1) Gyttja contenant du silt argilo-sablonneux $(0-63 \mathrm{~cm})$

Le faciès supérieur est constitué principalement de gyttja brunolive et représente des conditions de sédimentation lacustre. Le haut de la section, saturé d'eau, rendait la gyttja très floculante.

Contenu en eau moyen (\%) : 90,16

Perte au feu moyenne (\%) : 19,26

2) Gyttja contenant de l'argile silto-sablonneuse $(63-67 \mathrm{~cm})$ Les sédiments de la zone intermédiaire sont composés de gyttja contenant de l'argile silto-sablonneuse. Ils ont une texture gélatineuse et une couleur brun foncé.

Contenu en eau moyen (\%) : 86,81

Perte au feu moyenne (\%) : 14,58

3) Argile silteuse $(67-80 \mathrm{~cm})$

La section basale de la carotte longue est une argile silteuse qui, de par sa nature très compacte, a quelque peu limité la pénétration du carottier. La couleur du sédiment est d'un gris-vert uniforme.

Contenu en eau moyen (\%) : 50,12

Perte au feu moyenne (\%) : 4,35

sée de sédiments marins fins (argile silteuse) recouverts de boues lacustres organiques (gyttja). Ces deux unités sont séparées par un contact d'isolation stratigraphiquement plus ou moins bien défini, qui signale l'émergence isostatique du bassin de la Mer postglaciaire de Tyrrell et qui laisse croire que l'interface entre la phase d'accumulation argileuse et le début de l'accumulation de gyttja n'a pas subi d'érosion. La carotte courte est entièrement composée de gyttja et représente la séquence supérieure (plus récente) de l'accumulation sédimentaire.

\section{BIOSTRATIGRAPHIE}

Les tableaux III et IV résument les zones biostratigraphiques et diatomifères. La figure 3 montre les abondances relatives des espèces, classées selon leur tolérance à la salinité. Cette variable environnementale a eu une grande influence sur les variations d'assemblages de diatomées dans le lac Kachishayoot.

L'analyse de groupement illustre les changements dans les assemblages fossiles. Les zones de changements les plus manifestes identifiées par cette analyse correspondent à celles identifiées par le programme RATEPOL (fig. 4), confirmant ainsi la délimitation des différents paléoenvironnements.

\section{ALCALINITÉ}

La courbe de l'alcalinité reconstituée montre une diminution continuelle des valeurs en trois phases ou zones distinctes (fig. 5) :

La première zone, située entre $65,0 \mathrm{~cm}$ et $52,0 \mathrm{~cm}$ (ou entre 4100 et 2950 ans cal. BP / $3600-2700{ }^{14} \mathrm{C} \mathrm{BP}$ ), montre

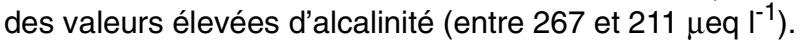

Le début de la deuxième zone coïncide avec une chute importante des valeurs (de 211 à $133 \mu \mathrm{eq}^{\mathrm{I}^{-1}}$ ). Cette zone est comprise entre $52,0 \mathrm{~cm}$ et $29,5 \mathrm{~cm}$ (ou entre 2950 et 1150 ans cal. BP / $2700-1200{ }^{14} \mathrm{C}$ BP). Les valeurs d'alcalinité varient entre 133 et $80 \mu \mathrm{eq} \mathrm{I}^{-1}$. 
TABLEAU IV

Aperçu de la succession des assemblages de diatomées dans le lac Kachishayoot

\section{Zone III $(0 \mathrm{~cm}-38 \mathrm{~cm})$}

Substrat : gyttja contenant du silt argilo-sablonneux

Formes de vie dominantes : taxons benthiques d'eau douce (épiphytiques)

Étendue de tolérance à la salinité : $0 \%$ (halophobe)

Espèces les plus importantes: Achnanthes altaica, A. kuelbsii, A. levanderi, A. marginulata, A. minutissima, A. pusilla, Brachysira brebissonnii, Cyclotella ocellata, Cymbella descripta, C. gaeumannii, C. hebridica, C. minutissima, Fragilaria pinnata, F. virescens var. exigua, Gomphonema parvulum, Navicula mediocris, Neidium ampliatum, Nitzschia fonticola, Pinnularia brauniana, Tabellaria flocculosa.

\section{Zone II a et b* $(38 \mathrm{~cm}-69 \mathrm{~cm})$}

Substrat : gyttja contenant de l'argile silto-sablonneuse $(69 \mathrm{~cm}-$ $63 \mathrm{~cm})$ et gyttja contenant du silt argilo-sablonneux $(63 \mathrm{~cm}-38$ $\mathrm{cm})$

Formes de vie dominantes : petits taxons benthiques d'eau douce (épiphytiques)

Étendue de tolérance à la salinité : 0-10\% (oligohalobe)

Espèces les plus importantes : Achnanthes cf. rosenstockii, Denticula cf. kuetzingii, Fragilaria brevistriata, F. elliptica, F. pinnata, F. pinnata var. intercedens, F. pseudoconstruens, Navicula modica.

${ }^{*}$ Cette zone a été subdivisée en deux sous-zones (a et b) à la profondeur de $51 \mathrm{~cm}$. La subdivision marque le début du déclin de la dominance de $F$. elliptica, ainsi que l'apparition progressive des espèces qui domineront la zone III.

\section{Zone I $(69 \mathrm{~cm}-80 \mathrm{~cm})$}

Substrat : argile silteuse

Formes de vie dominantes: taxons littoraux-marins benthiques (épipéliques et épiphytiques)

Étendue de tolérance à la salinité : 1-35\% (poly-mésohalobe)

Espèces les plus importantes: Cocconeis costata, Diploneis interrupta, Opephora marina, Paralia sulcata, Trachyneis aspera.

La dernière zone s'étend de $29,5 \mathrm{~cm}$ jusqu'à la surface (ou de 1150 ans cal. BP / $1200{ }^{14} \mathrm{C}$ BP à l'actuel). Elle est caractérisée par les plus faibles valeurs d'alcalinité (65 à $28 \mu \mathrm{eq}^{-1}$ ).

Les analogies établies entre les assemblages fossiles et le modèle de calibration du Québec-Labrador sont pauvres (fig. 5). Cela était prévisible puisque plusieurs niveaux présentent moins de $70 \%$ de similarité entre les espèces de la carotte et celles du modèle. Par contre, le test d'adéquation démontre que tous les assemblages sauf trois, se trouvent à l'intérieur de l'intervalle de confiance de $95 \%$. Ceci veut donc dire que le modèle de l'alcalinité traduit bien cette variable environnementale pour les assemblages de diatomées fossiles de cette séquence.

À la lumière de ces résultats, nous pouvons considérer que la reconstitution de l'alcalinité suit une tendance fiable (bon test d'adéquation). Par contre, les analogies entre les ensembles d'espèces étant pauvres, on ne doit pas accorder trop d'importance aux valeurs reconstituées.

D'après I'AC qui a été appliquée aux reconstitutions d'alcalinité et aux assemblages fossiles de diatomées (fig. 6), il semble que cette variable soit liée de près aux variations passées dans les assemblages diatomifères, puisque le coefficient de corrélation est de 0,92. L'alcalinité, ou des variables reliées de près à l'alcalinité (carbone inorganique dissous, $\mathrm{Ca}, \mathrm{Mg}, \mathrm{pH}$ ), aurait donc influencé de façon importante la distribution des espèces de diatomées au cours de l'histoire postglaciaire du lac Kachishayoot.

\section{DISCUSSION}

La séquence sédimentaire de la carotte longue semble démontrer que le processus d'isolation d'un bassin lacustre se déroule en trois phases : une phase franchement marine, lorsque le bassin communique avec la mer à tous les stades de la marée ; une phase de transition, quand le bassin à ses limites perd son contact régulier avec la mer, ce qui engendre des conditions d'eau saumâtre; et une phase d'eau douce, lorsque les invasions marines du bassin cessent. $\mathrm{Ce}$ processus d'isolation ressort bien de l'analyse sédimentaire et dans les assemblages de diatomées fossiles.

La section argileuse renferme un ensemble diatomifère dominé par des espèces périphytiques (Cocconeis costata, Diploneis interrupta, Opephora marina, Petroneis marina, Plagiogramma staurophorum, Rhabdonema minuta, Trachyneis aspera). L'espèce tychoplanctonique Paralia sulcata domine largement l'assemblage (<50\%) entre les niveaux 72 et $70 \mathrm{~cm}$ (fig. 3). Ce taxon est reconnu pour son écologie mal définie (McQuoid et Hobson, 1998). On rapporte sa présence autant dans les estuaires (Bérard-Thérriault et al., 1999), dans les marécages de la mer Baltique (Simonsen, 1962), que dans les 100 premiers mètres de surface de la mer de Norvège (Karpuz et Schrader, 1990) et dans les chenaux de marée de l'estuaire de la rivière Hudson (Weiss et al., 1978).

Si l'on se fie aux observations que rapporte Zong (1997b) sur les bassins d'isolation des côtes de la Grande-Bretagne, la dominance de $P$. sulcata serait le résultat de conditions favorables à cette espèce lors d'un épisode d'ingression marine. Selon Zong (1997b), P. sulcata serait avantagée par des conditions de grande variation de salinité, par des eaux agitées et troubles et par la présence d'un sédiment fin, riche en matière organique. Cette dernière caractéristique n'a toutefois pu être confirmée par l'analyse de la perte au feu des sédiments au contact d'isolation du lac Kachishayoot (PAF > que $5 \%$ ), probablement en raison de la nature instable du milieu côtier (la dynamique tidale et la présence de glace marine, par exemple). Par contre, la présence de graines de Potamogeton (macrophyte aquatique) a été signalée dans la séquence d'argile silteux (L. Miousse, communication personnelle), signalant ainsi la présence d'une végétation aquatique dans la région côtière hudsonienne de l'époque. Ces macrophytes sont caractéristiques des eaux calmes à modérément agitées et peu profondes (Porsild et Cody, 1980 ) et sont actuellement présents dans plusieurs régions 


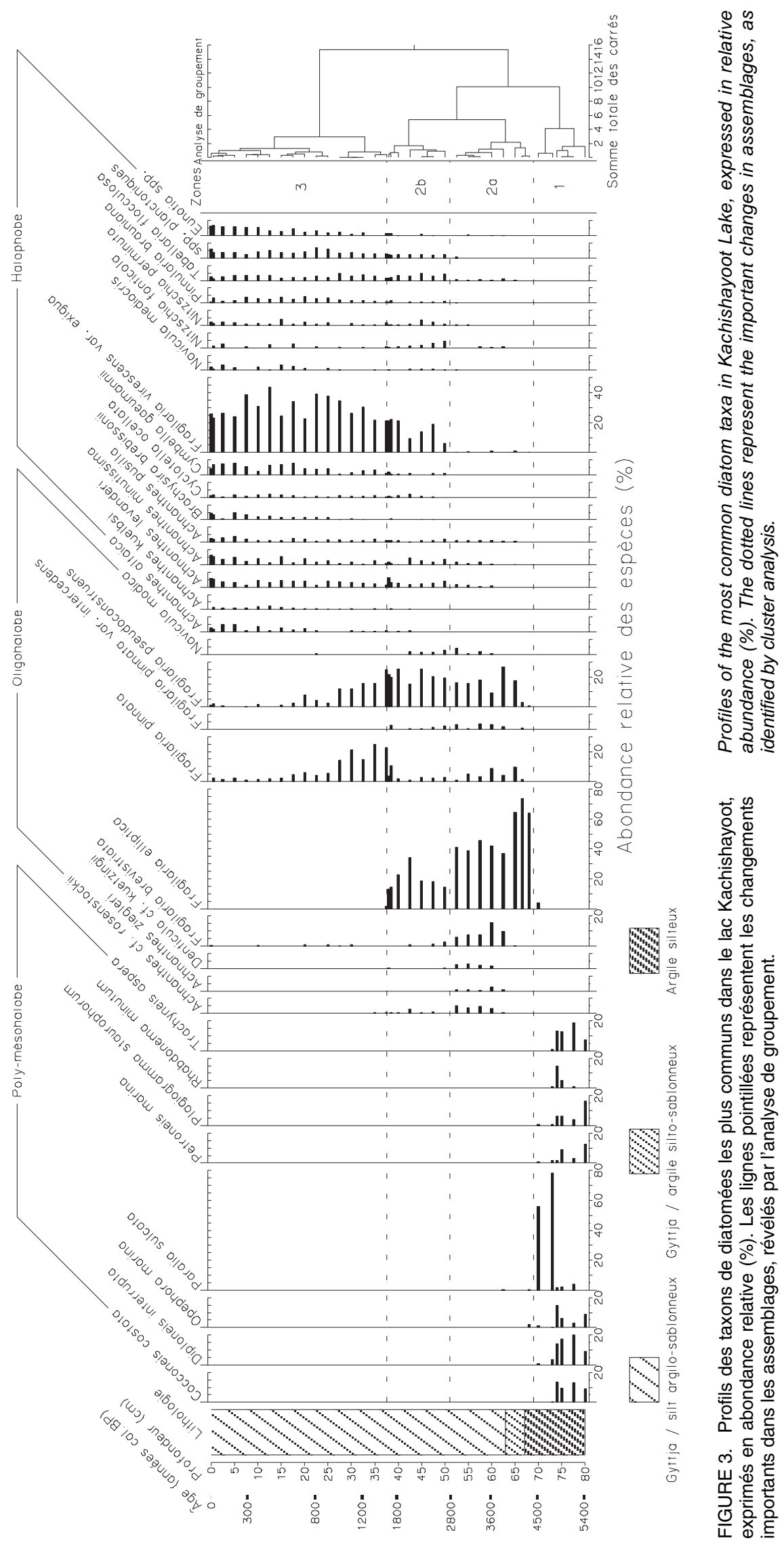

Géographie physique et Quaternaire, 55(1), 2001 


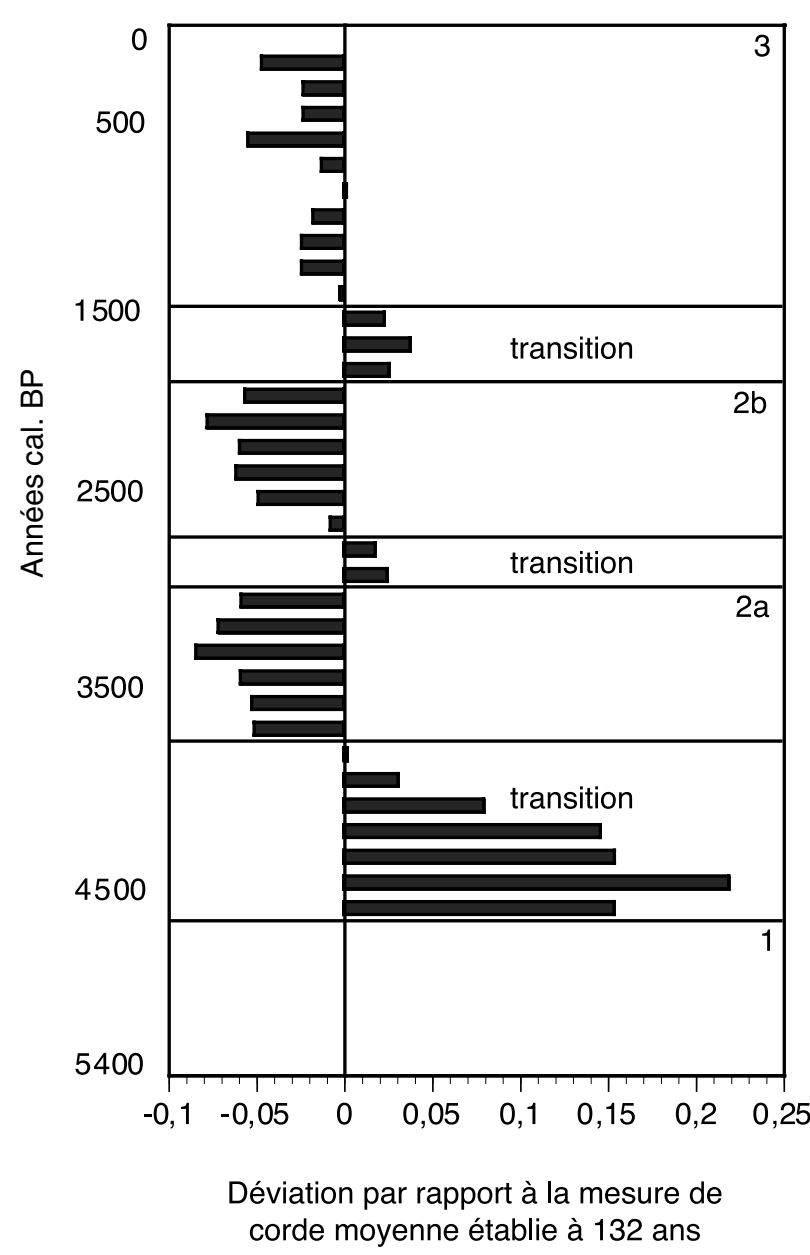

FIGURE 4. Taux de changement dans les assemblages de diatomées (intervalles de 132 ans), exprimés par une déviation par rapport à la mesure de corde moyenne. À droite, les chiffres correspondent aux zones identifiées par l'analyse de groupement de la figure 3 .

Rate of change in the diatom assemblages (per 132 years), expressed as deviation from the mean chord distance. The numbers on the right correspond to the zones identified by the cluster analysis in Figure 3.

nordiques du Québec et du Canada. Leur présence marque une source d'eau douce à proximité du site à cette époque.

En Norvège, la présence de $P$. sulcata dans les sédiments côtiers a été associée à des épisodes de climat relativement chaud, comme l'Allerød et l'Holocène (Stabell, 1980, 1985, 1986 ; Kjemperud, 1981 ; Lie et al., 1983 ; Krzywinski et Stabell, 1984 ; Stabell et Lange 1990). D'autres études (Conover, 1956 ; Karpuz et Schrader, 1990) semblent aussi indiquer que $P$. sulcata croît mieux à des températures plus chaudes.

Selon Richard (1979), la région du nord-est de la Jamésie aurait connu son optimum climatique postglaciaire entre 6500 et $2700{ }^{14} \mathrm{C}$ BP environ. L'analyse des restes chitineux de chironomides préservés dans la séquence sédimentaire du lac Kachishayoot confirme que les températures estivales de l'eau douce pour la période de 4000 à 3000 ans cal. BP $\left(3600-2700{ }^{14} \mathrm{C} \mathrm{BP}\right)$ étaient d'environ 4 à 8 degrés plus élevées qu'à l'actuel (K. Swadling, communica- tion personnelle). Les dates obtenues pour l'intervalle de 72 à $70 \mathrm{~cm}$ se trouvent à l'intérieur de la période caractérisée par des températures plus chaudes que la deuxième moitié de la période postglaciaire au Québec nordique (les 3000 dernières années, environ). Il est donc probable que ces températures chaudes aient favorisé le développement de $P$. sulcata lors de la phase marine terminale dans la région.

À la charnière entre les zones 1 et 2 (à $69 \mathrm{~cm}$ ), l'argile est remplacée par de la gyttja contenant de l'argile silto-sablonneuse. À ce niveau, $P$. sulcata cède brusquement la place à un assemblage composé presque essentiellement de petites espèces de Fragilaria alcaliphiles ( $F$. brevistriata, F. elliptica, $F$. pinnata, F. pinnata var. intercedens, F. pseudoconstruens). Ces dernières composent entre $45 \%$ et $93 \%$ du total des assemblages des niveaux compris entre $69 \mathrm{~cm}$ et $38 \mathrm{~cm}$. Ces espèces sont considérées comme étant opportunistes, bien adaptées à des conditions d'oligotrophie et de courtes saisons de croissance. Elles sont aussi qualifiées de pionnières parce qu'elles sont abondantes au cours des phases initiales de l'évolution des lacs et des épisodes de retrait des eaux marines (voir par exemple, Stabell, 1985 ; Pienitz et al., 1991 ; Zong, 1997a ; Seppä et Tikkanen, 1998). Parce que ces espèces semblent tolérer de grands écarts de salinité, on les dit indicatrices de l'isolation des bassins de la mer.

Dans les lacs depuis peu déglacés ou isolés d'une mer postglaciaire, les nutriments proviennent en grande partie du lessivage du till ou des argiles marines. Ce phénomène libère des quantités importantes d'éléments inorganiques et d'ions majeurs (carbone inorganique dissous, $\mathrm{Ca}, \mathrm{SiO}_{2}$ ) et entraîne du même coup une hausse de la conductivité et du $\mathrm{pH}$ (Stabell, 1985). En effet, la courbe d'alcalinité reflète ce phénomène : durant la phase lacustre initiale, l'alcalinité est très élevée, puis les valeurs diminuent graduellement à mesure que les ions majeurs sont éliminés par le recyclage des eaux. Le lac a atteint son taux actuel d'alcalinité (autour de $50 \mu \mathrm{eq} \mathrm{I}^{-1}$ ) vers 1000 ans cal. BP (1100 $\left.{ }^{14} \mathrm{C} \mathrm{BP}\right)$.

La courbe de l'alcalinité tracée à partir des diatomées montre un écart important de valeurs au cours de la période postglaciaire (entre 28,6 et $267,0 \mu \mathrm{eq} \mathrm{I}^{-1}$ ) (fig. 5). Les taux sont très élevés au moment de l'isolation du bassin, puis montrent une chute de la concentration lors de la phase lacustre initiale. Comme il a été mentionné plus haut, ceci reflète les changements rapides survenus dans le bassin versant et la trophie de l'eau du lac Kachishayoot (qui passait d'un état riche à un état pauvre en nutriments). La baisse de l'alcalinité coïncide avec la disparition de quelques taxons de diatomées alcaliphiles, comme Cocconeis costata, Diploneis interrupta et Paralia sulcata dans un premier temps (autour de $70 \mathrm{~cm}$ ), suivis d'Achnanthes cf. rosenstockii, Fragilaria brevistriata et $F$. pinnata var. intercedens.

Les tendances observées dans l'alcalinité du lac Kachishayoot correspondent à celles d'autres lacs nordiques : la courbe de l'alcalinité établie à partir des diatomées pour le lac Hope Simpson, au Labrador (Allaire, 1997), montre la même tendance, mais sur une plus longue période. Cette tendance d'une perte progressive d'alcalinité jumelée à l'augmentation correspondante du taux de carbone organique 


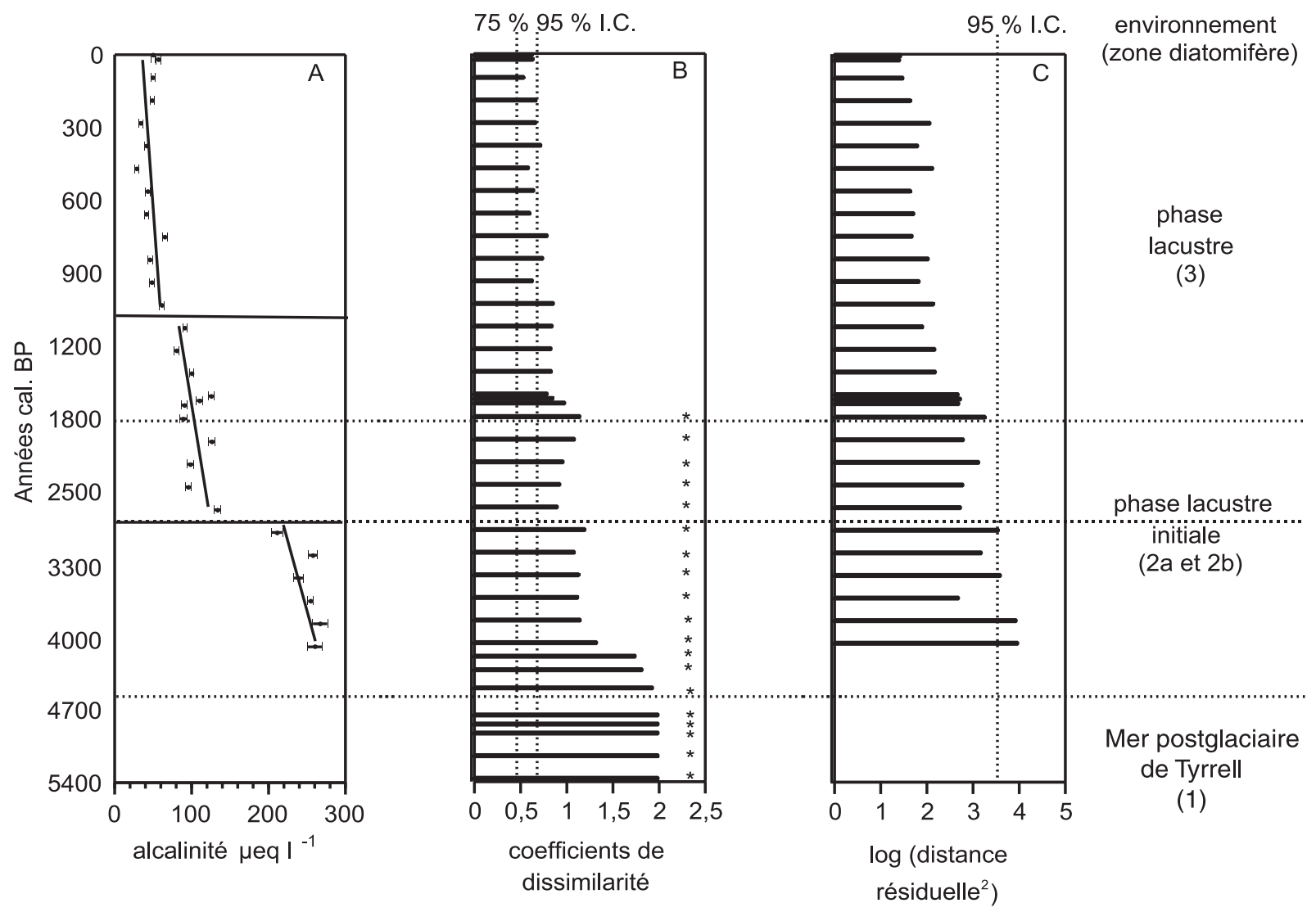

FIGURE 5. Analyses statistiques des assemblages fossiles de diatomées par rapport au modèle de l'alcalinité. Les lignes horizontales délimitent les changements d'environnement. Les astérisques indiquent les niveaux où moins de $70 \%$ des assemblages fossiles de diatomées étaient représentés dans le modèle de calibration. A) Reconstitution de l'alcalinité (points) et de l'étendue des erreurs possibles (lignes) en $\mu$ eq $\mathrm{I}^{-1}$. B) Analogies entre les assemblages de diatomées dans la carotte de sédiments et le modèle de calibration. Les échantillons dont les coefficients de dissimilarité (CD) excédent la limite de l'intervalle de confiance (IC) de $95 \%$ sont considérés comme n'ayant pas d'analogie avec l'ensemble de calibration. C) Analogies d'alcalinité des assemblages de diatomées dans la carotte de sédiments. Les échantillons dont les log (distances résiduelles au carré) excèdent la limite de confiance de $95 \%$ sont considérés comme de pauvres analogues d'alcalinité.

dissous (Saulnier-Talbot, 2000) a aussi été observée par Engstrom et al. (2000) dans une série de lacs récemment déglacés du sud de l'Alaska.

Le climat et la végétation peuvent aussi indirectement jouer un rôle important dans les concentrations d'alcalinité d'un lac (Laing et al., 1999b ; Ponader, 2000). Un climat plus chaud peut entraîner une augmentation de la productivité primaire ou encore favoriser la dégradation du pergélisol, ce qui faciliterait l'apport d'acides humiques et fulviques (s'il s'en trouve) dans l'eau du lac, abaissant ainsi son alcalinité (Rühland et Smol, 1998).

Selon nos observations litho- et biostratigraphiques, l'isolation du bassin lacustre (ou la perte d'un contact régulier avec la mer) serait survenue près du niveau de $69 \mathrm{~cm}$ vers
Statistical analysis of fossil diatom assemblages in relation to the alkalinity model. The horizontal lines delimit environmental changes. The asterisks indicate the levels where less than $70 \%$ of the fossil assemblage was present in the calibration model. A) Reconstruction of alkalinity (points) and the possible error (lines) in $\mu$ eq $r^{-1}$. B) Analogs between the diatom assemblages from the sediment core and the calibration model. The samples with a dissimilarity coefficient (DC) exceeding the confidence interval $(\mathrm{Cl})$ limit of $95 \%$ are considered as having no analogies within the the calibration set. C) Alkalinity analogies of the diatom assemblages from the sediment core. The samples with a log squared residual distance exceeding the limit confidence interval of $95 \%$ would be considered as poor alkalinity analogs.

4500 ans cal. BP/4000 ans ${ }^{14} \mathrm{C}$ BP. Aucun indice ne laisse croire que cette partie de la séquence ait subi d'érosion. L'absence de preuves de l'existence d'une séquence d'isolation non préservée confirme donc que la séquence est complète et que l'isolation a été rapide. Selon la sédimentologie et les dates extrapolées, la durée de la période d'isolation est estimée à environ 1000 ans.

La proximité des îles Manitounuk (fig. 2) permet d'intégrer les résultats de la présente étude à la courbe d'émersion postglaciaire dressée pour cet archipel côtier par Allard et Tremblay (1983). Entre 60 et 140 mètres d'altitude, leur courbe ne comprend pas de datations. La figure 7 illustre le contact d'isolation du lac Kachishayoot (100 m d'altitude) superposé à la courbe d'émersion. Ce point fait dévier la courbe légèrement vers le haut, impliquant que le taux de 


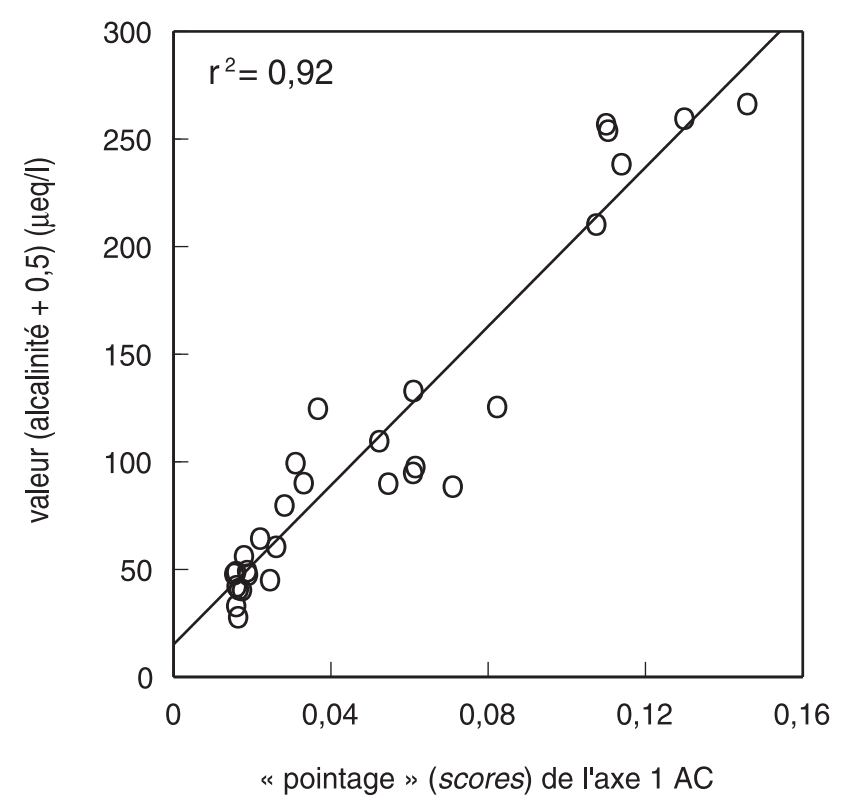

FIGURE 6. Relation entre les « pointages » (scores) de l'axe AC 1 pour les assemblages de diatomées dans la carotte et les valeurs reconstituées d'alcalinité. Les cercles représentent les niveaux pour lesquels une valeur d'alcalinité a été calculée.

Relationship between CA axis scores for diatom assemblages in the sediment core and reconstructed alkalinity values. The circles represent the levels for which an alkalinity value was inferred.

relèvement isostatique aurait été plus rapide au cours de la période postglaciaire initiale. Une datation du niveau situé à $69 \mathrm{~cm}$ permettrait de préciser l'allure de la courbe.

La dernière section de la séquence sédimentaire du lac Kachishayoot $(38-0 \mathrm{~cm}$ ) est composée de gyttja contenant du silt argilo-sablonneux où l'assemblage diatomifère est dominé par des espèces pennales benthiques comme Achnanthes levanderi, A. minutissima, Cymbella gaeumannii, Fragilaria pinnata, F. virescens var. exigua et l'espèce tychoplanctonique Tabellaria flocculosa. Ces espèces ont été observées en grand nombre dans les assemblages actuels de plusieurs autres lacs du Québec nordique, situés de part et d'autre de la limite des arbres (Fallu et Pienitz, 1999 ; Ponader, 2000). Des assemblages similaires ont été recensés par Allaire (1997) au Québec-Labrador, par Pienitz et al. $(1995,1997)$ dans des lacs situés à la limite des arbres dans les Territoires du Nord-Ouest et au Yukon, par GregoryEaves (1998) en Alaska, par Weckström et al. (1997) en Fennoscandie et par Laing et al. (1999a) dans la toundra de la Sibérie orientale. Ces auteurs soulignent l'existence indéniable d'une flore diatomifère typique des régions circumpolaires arctiques à la limite des arbres.

Cette dernière section représente la phase lacustre proprement dite du lac Kachishayoot. Le passage d'une flore diatomifère pionnière à une flore dominée par des espèces typiquement subarctiques montre que le lac a atteint une certaine maturité et une stabilité depuis un peu plus de 1500 ans (cal. BP). La microflore de cette section est essentiellement composée d'espèces circumneutres à acidophiles. Fragilaria virescens var. exigua en est l'espèce

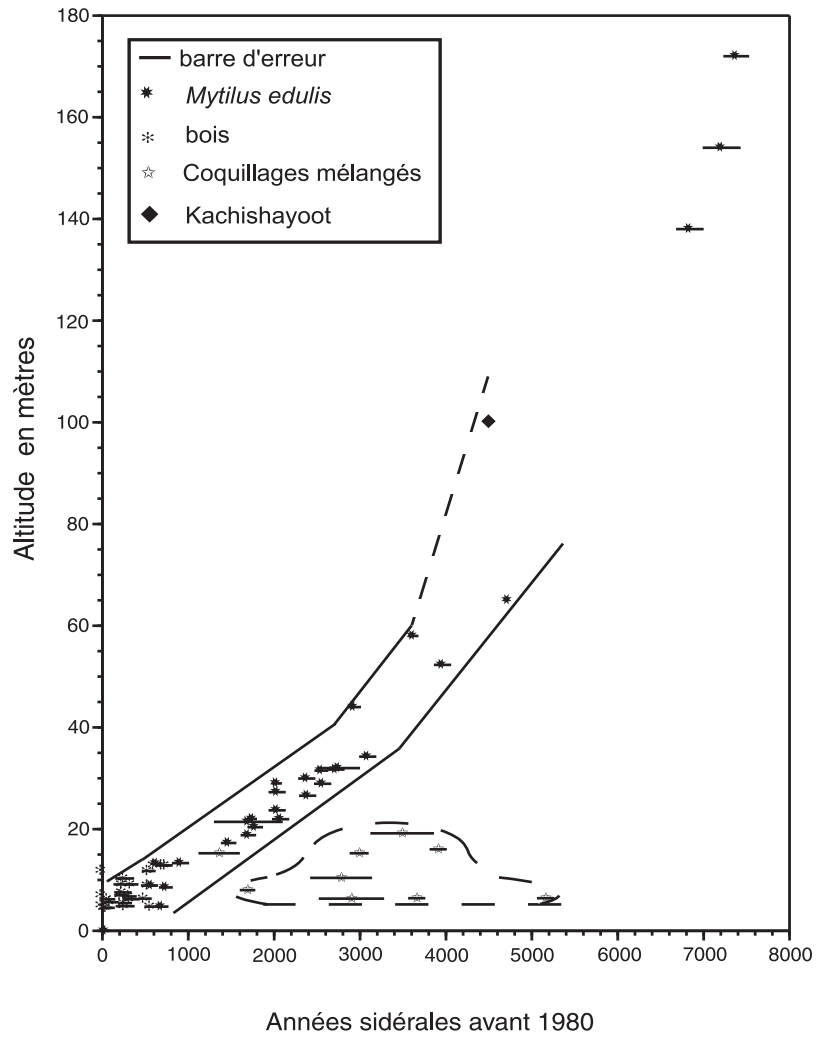

FIGURE 7. Courbe de relèvement isostatique de la région côtière des îles Manitounuk, en Hudsonie, au Québec (tiré de Allard et Tremblay, 1983). Le losange noir représente l'âge mesuré à l'aide des datations SMA (tabl. II) identifiant le contact d'isolation marine/lacustre dans le bassin du lac Kachishayoot. Ce niveau se trouve présentement à une altitude de 100 mètres.

Postglacial emergence curve for the Manitounuk Islands as illustrated by ${ }^{14} \mathrm{C}$ dates (from Allard et Tremblay, 1983). The years are expressed in Cal yr before 1980. The black lozenge represents the age inferred by the dates obtained by AMS (Tabl. II) for the marine/lacustrine isolation contact in Kachishayoot Lake. This level is presently situated at an altitude of 100 metre s above sea-level.

dominante, avec entre $21 \%$ et $43 \%$ d'abondance relative. II s'agit d'une espèce périphytique qui préfère les eaux circumneutres à légèrement acides (Krammer et Lange-Bertalot, 1991a ; Pienitz et Smol, 1993 ; Pienitz et al., 1999) et qui domine dans plusieurs lacs du Nord québécois (Fallu et Pienitz, 1999 ; Ponader, 2000). Les autres espèces les plus communes des assemblages sont en majorité circumneutres (Achnanthes altaica, A. kuelbsi, A. levanderi, A. minutissima, A. pusilla, Cyclotella ocellata, Navicula mediocris) et acidophiles (Brachysira brebissonii, Cymbella gaeumannii, Pinnularia brauniana, Tabellaria flocculosa) (fig. 3), ce qui confirme les préférences de $F$. virescens var. exigua.

\section{CONCLUSION}

L'analyse des diatomées fossiles dans la séquence sédimentaire du lac Kachishayoot s'est avérée un outil précieux pour la reconstitution des changements environnementaux postglaciaires survenus dans la région de Kuujjuaraapik: côte en émersion, retrait de la mer, formation d'un lac. 
Les séquences sédimentaires et biostratigraphiques décrites plus haut, ainsi que les résultats des datations SMA permettent d'affirmer que la carotte prélevée dans le lac Kachishayoot couvre la totalité de la période suivant le retrait de la Mer postglaciaire de Tyrrell dans la région d'étude (de 5400 ans cal. BP ou 5000 ans ${ }^{14} \mathrm{C}$ environ à aujourd'hui).

Comparés à la courbe d'émersion dressée pour les îles Manitounuk, les résultats semblent indiquer un taux de relèvement isostatique plus rapide pour la région entre 4500 et 3500 ans cal. BP environ, faute de données à ces altitudes élevées. Cet intervalle correspond à la période de l'isolation du bassin du lac Kachishayoot. Toutefois, des datations supplémentaires du contact d'isolation dans le lac ainsi que dans quelques sites environnants situés à différentes altitudes permettraient de préciser les variations dans la courbe de relèvement isostatique de la région, surtout pour l'époque antérieure à 5000 ans cal. BP.

\section{REMERCIEMENTS}

Ce projet a bénéficié d'une subvention de recherche du Fonds pour la Formation des Chercheurs et l'Aide à la Recherche accordée à Reinhard Pienitz. Nous tenons à remercier John A. E. Gibson, Karin Ponader et Kerrie M. Swadling pour leur aide sur le terrain, ainsi que le Centre d'études nordiques pour son soutien logistique. Nous sommes aussi très reconnaissants envers Tamsin E. Laing pour ses commentaires et conseils qui ont permis d'améliorer le manuscrit. Nous remercions les habitants de KuujjuaraapikWhapmagoostui pour l'utilisation de leur réseau de chemins VTT. Merci également à Michel Poulin et un lecteur anonyme pour leur lecture critique du manuscrit.

\section{RÉFÉRENCES}

Allaire, N., 1997. Relation entre les assemblages de diatomées et les variables environnementales de 70 lacs du Québec-Labrador et résultats préliminaires d'une étude paléolimnologique du lac Hope Simpson. Thèse de maîtrise, Université Laval, 161 p.

Allard, M. et Séguin, M.K., 1987. Le pergélisol au Québec nordique : bilan et perspectives. Géographie physique et Quaternaire, 41 : 141-152.

Allard, M. et Tremblay, G., 1983. La dynamique littorale des îles Manitounuk durant l'Holocène. Zeitschrift für Geomorphologie, Supplement Band 47 : 61-95.

Barber, H.G. et Haworth, E.Y., 1981. A Guide to the Morphology of the Diatom Frustule. Freshwater Biological Association Scientific Publication 44, 113 p.

Bégin, Y., Bérubé D. et Grégoire, M., 1993. Downward migration of coastal conifers as a response to recent land emergence in eastern Hudson Bay, Québec. Quaternary Research, $40:$ 81-88.

Bérard-Thérriault, L., Poulin, M. et Bossé, L., 1999. Guide d'identification du phytoplancton marin de l'estuaire et du golfe du St-Laurent incluant également certains protozoaires. Publication spéciale canadienne des sciences halieutiques et aquatiques $128,387 p$.

Bilodeau, G., De Vernal, A., Hillaire-Marcel, C. et Josenhans, H., 1990. Postglacial paleoceanography of Hudson Bay: Stratigraphic, microfaunal, and palynological evidence. Canadian Journal of Earth Sciences, 27 : 946-963.

Birks, H.J.B., Juggins, S. et Line, J.M., 1990. Lake surface-water chemistry reconstruction from palaeoecological data, p. 301-311. In B. J. Mason, édit., The Surface Waters Acidification Program. Cambridge University Press, $522 \mathrm{p}$.
Campeau, S., Pienitz, R. et Héquette, A., 1999a. Diatoms from the Beaufort Sea coast, southern Arctic Ocean (Canada). Bibliotheca Diatomologica 42, J. Cramer, Berlin/Stuttgart, $244 \mathrm{p}$.

1999b. Diatoms as quantitative paleodepth indicators in coastal areas of the southeastern Beaufort Sea, Arctic Ocean. Palaeogeography, Palaeoclimatology, Palaeoecolgy, 146 : 67-97.

Campeau, S., Héquette, A. et Pienitz, R., 2000. Late Holocene diatom biostratigraphy and sea-level changes in the southeastern Beaufort Sea. Canadian Journal of Earth Sciences, 37 : 63-80.

Conover, S.A.M., 1956. Oceanography of Long Island Sound, 1952-1954. IV. Phytoplankton. Bulletin of the Bingham Oceanographic Collection, $15: 62-112$.

Engstrom, D.R., Fritz, S.C., Almendinger, J.E. et Juggins, S., 2000. Chemical and biological trends during lake evolution in recently deglaciated terrain. Nature, $408: 161-166$.

Environnement Canada, 1999. Site internet. http://www.cmc.ec.gc.ca/climat

Fallu, M.-A., Allaire, N. et Pienitz, R., 2000. Freshwater Diatoms from Northern Québec and Labrador (Canada). Bibliotheca Diatomologica, J. Cramer Berlin/Stuttgart, vol. 45, $200 \mathrm{p}$.

Fallu, M.-A. et Pienitz, R., 1999. Diatomées lacustres de Jamésie-Hudsonie (Québec) et modèle de reconstitution des concentrations de carbone organique dissous. Écoscience, $6: 603-620$.

Gibson, J.A.E., Vincent, W.F. et Pienitz, R., 2001. Hydrologic control and diurnal photobleaching of CDOM in a subarctic lake. Archiv für Hydrobiologie, 152 : 143-159.

Gregory-Eaves, I., 1998. Reconstructing climatic and environmental trends in Alaska using diatom-based paleolimnology. Thèse de maîtrise, Queen's University, $165 \mathrm{p}$.

Grimm, E.C., 1987. Coniss : A FORTRAN 77 program for stratigraphically constrained cluster analysis by the method of incremental sum of squares. Computers and Geosciences, 13 : 13-35.

1991. TILIA, version 2.00, Illinois State Museum Research and Collections Center, Springfield, Illinois.

1991-1993. TILIAGRAPH, version 1.25, Illinois State Museum Research and Collections Center, Springfield, Illinois.

Juggins, S. et ter Braak, C.J.F., 1992. CALIBRATE - a program for speciesenvironment calibration by [weighted averaging] partial least squares regression. Environmental Change Research Center, University College, London.

Karpuz, N.K. et Schrader, H., 1990. Surface sediment diatom distribution and Holocene paleotemperature variations in the Greenland, Iceland and Norwegian Sea. Paleoceanography, 5 : 557-580.

Kjemperud, A., 1981. Diatom changes in sediments of basins possessing marine/lacustrine transitions in Frosta, Nord-Trøndelag, Norway. Boreas, $10: 27-38$.

Krammer, K. et Lange-Bertalot, H., 1986. Bacillariophyceae. 1. Teil : Naviculaceae. H. Ettl, G. Gärtner, J. Gerloff, H. Heynig et D. Mollenhauer, édit., Süßwasserflora von Mitteleuropa, Band 2/1. Gustav Fischer Verlag, Stuttgart/New York, $876 \mathrm{p}$.

1988. Bacillariophyceae. 2. Teil : Bacillariaceae, Epithemiaceae, Surirellaceae. H. Ettl., J. Gerloff, H. Heynig et D. Mollenhauer, édit., Süßwasserflora von Mitteleuropa, Band 2/2. Gustav Fischer Verlag, Stuttgart/New York, $596 \mathrm{p}$.

1991a. Bacillariophyceae. 3. Teil : Centrales, Fragilariaceae, Eunotiaceae. H. Ettl., J. Gerloff, H. Heynig et D. Mollenhauer, édit., Süßwasserflora von Mitteleuropa, Band 2/3. Gustav Fischer Verlag, Stuttgart/Jena, $576 \mathrm{p}$.

1991b. Bacillariophyceae. 4. Teil : Achnanthaceae, Kritische Ergänzungen zu Navicula (Lineolatae) und Gomphonema. H.Ettl., G. Gärtner, J. Gerloff, H. Heynig et D. Mollenhauer, édit., Süßwasserflora von Mitteleuropa, Band 2/4. Gustav Fischer Verlag, Stuttgart/Jena, 437 p.

Krzywinski, K. et Stabell, B., 1984. Late Weichselian sea level changes at Sotra, Hordaland, western Norway. Boreas, 13 : 159-202.

Laing, T.E., Pienitz, R. et Smol, J.P., 1999a. Freshwater diatom assemblages from 23 lakes located near Norilsk, Siberia: A comparison with assemblages from other circumpolar treeline regions. Diatom Research, $14: 285-305$. 
Laing, T.E., Rühland, K. et Smol, J.P., 1999b. Past environmental and climatic changes related to tree-line shifts inferred from fossil diatoms from a lake near the Lena River Delta, Siberia. The Holocene, 9 : 547-557.

Laird, K.R., Fritz, S.C. et Cumming, B.F., 1998. A diatom-based reconstruction of drought intensity, duration, and frequency from Moon Lake, North Dakota: A sub-decadal record of the last 2300 years. Journal of Paleolimnology, $19: 161-179$.

Lie, S. E., Stabell, B. et Mangerud, J., 1983. Diatom stratigraphy related to Late Weichselian sea-level changes in Sunnmore, Western Norway. Norges Geologiske Undersokelse, 380 : 203-219.

Line, J.M. et Birks, H.J.B., 1994. RATEPOL, version 0.8. Non publié.

McQuoid, M.R. et Hobson, L.A., 1998. Assessment of paleoenvironmental conditions on southern Vancouver Island, British Columbia, Canada, using the marine tychoplancter Paralia sulcata. Diatom Research, $13: 311-321$

Overpeck, J.T., Webb III, T. et Prentice, I.C., 1985. Quantitative interpretation of fossil pollen spectra: Dissimilarity coefficients and the method of modern analogs. Quaternary Research, 23 : 87-108.

Palmer, A.J.M. et Abbott, W.H., 1986. Diatoms as indicators of sea-level change, p. 457-487. In O. Van de Plassche, édit., Sea-Level Reseach : A manual for the collection and evaluation of data. Geo Books, Norwich, 600 p.

Payette, S., 1983. The forest tundra and present tree-lines of the northern Québec-Labrador Peninsula. In P. Morisset. et S. Payette, édit., Tree-line ecology: Proceedings of the northern Québec-Labrador treeline conference. Université Laval, Québec, Collection Nordicana, 47 : 3-23.

Pienitz, R., Lortie, G. et Allard, M., 1991. Isolation of lacustrine basins and marine regression in the Kuujjuaq area (northern Québec), as inferred from diatom analysis. Géographie physique et Quaternaire, $45: 155-174$.

Pienitz, R. et Smol, J.P., 1993. Diatom assemblages and their relationship to environmental variables in lakes from the boreal forest-tundra ecotone near Yellowknife, Northwest Territories, Canada. Hydrobiologia, 269/270 : 391-404.

Pienitz, R., Smol, J.P et Birks, H.J.B., 1995. Assessment of freshwater diatoms as quantitative indicators of past climatic change in the Yukon and Northwest Territories, Canada. Journal of Paleolimnology, 13 : 21-49.

Pienitz, R., Smol, J.P. et Lean, D.R.S., 1997. Physical and chemical limnology of 24 lakes located between Yellowknife and Contwoyto, Northwest Territories (Canada). Journal canadien des sciences halieutiques et aquatiques, $54: 347-358$.

Pienitz, R., Smol, J.P. et MacDonald, G.M., 1999. Paleolimnological reconstruction of Holocene climatic trends from two boreal treeline lakes, Northwest Territories, Canada. Arctic, Antarctic and Alpine Research, 31 : 82-93.

Ponader, K., 2000. Analyse paléolimnologique d'un lac subarctique à la limite des arbres, Québec nordique, basée sur les assemblages fossiles de diatomées. Thèse de maîtrise, Université Laval, $85 \mathrm{p}$.

Porsild, A.E. et Cody, W.J., 1980. Vascular Plants of Continental Northwest Territories, Canada. National Museums of Canada, Ottawa, 668 p.

Richard, P.J. H., 1979. Contribution à I'histoire postglaciaire de la végétation au nord-est de la Jamésie, Nouveau-Québec. Géographie physique et Quaternaire, 33 : 93-112.

Rühland, K. et Smol, J.P., 1998. Limnological characteristics of 70 lakes spanning Arctic treeline from Coronation Gulf to Great Slave Lake in the Central Northwestern Territories, Canada. Internationale Revue der gesamten Hydrobiologie, 83 : 183-203.

Saulnier-Talbot, É., 2000. Succession lacustre et variations limnologiques holocènes du lac Kachishayoot, Québec nordique, inférées par les assemblages fossiles de diatomées. Thèse de maîtrise, Université Laval, $105 \mathrm{p}$.

Seppä, H. et Tikkanen, M., 1998. The isolation of Kruunuvuorenlampi, southern Finland, and implications for Holocene shore displacement models of the Finnish south coast. Journal of Paleolimnology, 19 : 385398.
Simonsen, R., 1962. Untersuchungen zur Systematik und Ökologie der Bodendiatomeen der westlichen Ostsee. Internationale Revue der Gesamten Hydrobiologie, Systematische Beihefte I, 144 p.

Snoeijs, P., édit., 1993. Intercalibration and distribution of diatom species in the Baltic Sea, Vol. 1. Baltic Marine Biologists Publication 16a, Opulus Press, Uppsala, $129 \mathrm{p}$.

Snoeijs, P. et Balashova, N., édit., 1998. Intercalibration and distribution of diatom species in the Baltic Sea, Vol. 5. Baltic Marine Biologists Pubublication 16e, Opulus Press, Uppsala, 144 p.

Snoeijs, P. et Kasperoviciene, J., édit., 1996. Intercalibration and distribution of diatom species in the Baltic Sea, Vol. 4. Baltic Marine Biologists Publication 16d, Opulus Press, Uppsala, $126 \mathrm{p}$.

Snoeijs, P. et Potapova, M., édit., 1995. Intercalibration and distribution of diatom species in the Baltic Sea, Vol. 3. Baltic Marine Biologists Publication 16c, Opulus Press, Uppsala, $126 \mathrm{p}$.

Snoeijs, P. et Vilbaste, S. édit, 1994. Intercalibration and distribution of diatom species in the Baltic Sea, Vol. 2. Baltic Marine Biologists Publication 16b, Opulus Press, Uppsala, $125 \mathrm{p}$.

Stabell, B., 1980. Holocene shorelevel displacement in Telemark, southern Norway. Norsk Geologisk Tidsskrift, 60 : 71-81.

1985. The development and succession of taxa within the diatom genus Fragilaria Lyngbye as a response to basin isolation from the sea. Boreas, $14: 273-286$.

1986. A diatom maximum horizon in upper Quaternary deposits. Geologische Rundschau, 75 : 175-184.

Stabell, B. et Lange, C.B., 1990. Diatom assemblages in upper Quaternary sediments from the south-eastern Norwegian Sea, core P 76-17. Beiheft Nova Hedwigia, $100: 289-300$.

Stuiver, M. et Reimer, P.J., 1993. Extended ${ }^{14} \mathrm{C}$ data base and revised CALIB $3.0{ }^{14} \mathrm{C}$ age calibration program. Radiocarbon, $35: 215-230$.

ter Braak, C.J.F., 1995. Ordination, p. 91-173. In R.H.G. Jongman, C.J.F. ter Braak et O.F.R. van Tongeren, édit., Data analysis in community and landscape ecology. Cambridge University Press, $299 \mathrm{p}$.

ter Braak, C.J.F. et Smilauer, P., 1998. CANOCO Reference manual and user's guide to Canoco for Windows : Software for canonical community ordination (version 4), Microcomputer Power, Ithaca, 352 p.

Veillette, J.J., 1994. Evolution and paleohydrology of glacial lakes Barlow and Ojibway. Quaternary Science Reviews, $13: 945-971$.

Vincent, J.-S. et Hardy, L., 1977. L'évolution et l'extension des lacs glaciaires Barlow et Ojibway en territoire québécois. Géographie physique et Quaternaire, $31:$ 357-372.

Weckström, J., Korhola, A. et Blom, T., 1997. The relationship between diatoms and water temperature in thirty subarctic Fennoscandian lakes. Arctic and Alpine Research, 29 : 75-92.

Weiss, D., Geitzenauer K. et Shaw, F.C., 1978. Foraminifera, diatom and bivalve distribution in recent sediments of the Hudson estuary. Estuarine and Coastal Marine Science, $7: 393-400$.

Wilson, S.E., Cumming, B.F. et Smol, J.P., 1996. Assessing the reliability of salinity inference models from diatom assemblages : an examination of a 219-lake data set from western North America. Canadian Journal of Fisheries and aquatic Sciences, $53: 1580-94$.

Witkowski, A., 1994. Recent and fossil diatom flora of the Gulf of Gdansk, Southern Baltic Sea: Origin, composition and changes of diatom assemblages during the Holocene. Bibliotheca Diatomologica, Band 28, J. Cramer, Berlin/Stuttgart, $312 \mathrm{p}$.

Zong, Y., 1997a. Mid- and late-Holocene sea-level changes in Roudsea Marsh, northwest England: A diatom biostratigraphical investigation. The Holocene, $7:$ 311-323.

1997b. Implications of Paralia sulcata abundance in Scottish isolation basins. Diatom Research, $12: 125-150$. 